\title{
A Systematic LT Coded Arrangement for Transmission Over Correlated Shadow Fading Channels in 802.11 Ad-hoc Wireless Networks
}

\author{
Hoang Anh Ngo, Tim Stevens, Robert G. Maunder and Lajos Hanzo \\ School of Electronics \& Computer Science, University of Southampton \\ Email: $\{$ han08r, tas07r, rm, lh\}@ecs.soton.ac.uk
}

\begin{abstract}
Systematic Luby Transform (SLT) codes constitute rateless codes, which are capable of adaptively adjusting their code rate depending on the channel quality without any explicit channel state information (CSI) at the transmitters. In this paper, we investigate the performance of an SLT coded wireless system communicating over a correlated fading channel within an 802.11b network. The simulation results show that the SLT coded system can achieve a $3 \mathrm{~dB}$ gain in term of fading deviation when fading correlation changes from $1 \mathrm{~Hz}$ to $5 \mathrm{~Hz}$. Moreover, it is found that a system employing an SLT code can provide similar performance to other rateless codes, namely the Luby Transform (LT) code and the Random Linear Fountain Code (RLFC). However, this level of performance is achieved with a reduced number of decoding operations. A $20 \%$ reduction is achieved when the standard deviation of the fading amplitude is $10 \mathrm{~dB}$ and the communication distance is $7 \mathrm{~m}$. Greater reductions are observed under more benign propagation conditions.

Index Terms - Systematic Luby Transform (SLT) code, rateless code, path-loss, correlated shadow fading, $\mathbf{8 0 2 . 1 1 b}$ wireless network.
\end{abstract}

\section{INTRODUCTION}

The 802.11 standard [1] defines a family of protocols that have been widely adopted. The standard specifies a wireless network capable of operating over a wide range of data rates, which is achieved through the selection of both the modulation scheme and the rate of the channel code employed [2]. Additionally, the standard supports two major modes of operation based on either a central infrastructure or on an ad-hoc configuration. The infrastructure based mode assigns additional responsibilities to the Access Point (AP) in order to coordinate channel access. By contrast, in the ad-hoc mode each station is a peer with an identical status, hence the access management is distributed amongst the Mobile Stations (MS) collaborating in the network. Since we wish to evaluate the achievable performance of application-layer Forward Error Coding (FEC) using a selection of rateless codes, we have opted for using an ad-hoc 802.11b network comprising two nodes and using an $11 \mathrm{Mbps}$ service operating at a carrier frequency of $2442 \mathrm{MHz}$. All other system parameters are summarised in Table I.

The signal amplitude received by an MS is affected by both the distance the signal has to propagate and the presence of any fading mechanism. Fading may be broadly categorised

The financial support of EPSRC, UK under the auspices of the UK-India Centre of Excellence in Wireless Communications as well as that of the EU under the Optimix project is gratefully acknowledged. as being either shadowing induced slow-fading or multipath induced fast-fading. The former is largely determined by the terrain through which the MS is moving and is modelled as having a distribution characterised by a log-normal probability density function.

Jakes' model [3], using the sum-of-sinusoids technique in conjunction with random phases was incorporated in the network simulator [4]. Due to the assumption that the received signal amplitude is constant over a packet, which is inherent in the network simulator's implementation, fast fading was not considered in this analysis. The path loss attributed to the distance between the pair of MSs considered was determined by the free-space model of Friis [5]

$$
\alpha(d)=G_{T} G_{R}\left(\frac{\lambda}{4 \pi d}\right)^{2},
$$

where the terms are defined in Table I. We positioned the MSs to achieve a predetermined received signal amplitude relative to a reference level.

The Systematic Luby Transform (SLT) code family was proposed in [6], which is an evolved version of the LT code [7] and it includes all the original source data in degree-one packets at the start of the LT-encoded stream. Also, in contrast to the hard-decision nature of a conventional LT code [7], the SLT code may be processed by both hard- and soft-decoding techniques. Therefore, the SLT code offers the possibility of a significant improvement in the achievable performance. The achievable soft-decoding performance was quantified in [6]. In this paper, we proposed that a FEC scheme be implemented at the application layer based on an SLT code for transmission over an $802.11 \mathrm{~b}$ wireless network. Compared to the classic physical-layer FEC schemes, which are typically incorporated in the hardware devices, the application-layer FECs provide a higher flexibility in customizing the code parameters, and therefore, they may be expected to improve the performance of the entire system. The paper's contributions are as follows:

1) The performance of the SLT coded application-layer is compared to those of other rateless benchmark codes, such as a Random Linear Fountain Code (RLFC) and a conventional LT code. Each coded system is characterised in terms of both the achievable Bit Error Ratio (BER) and the Block Error Ratio (BLER). The results are presented for channel conditions characterised by both the standard deviation of the shadow fading amplitude and the shadow fading frequency. 
TABLE I

THE MAJOR SIMULATIONS PARAMETERS

\begin{tabular}{|c|c|}
\hline Nodes & 2 \\
\hline Payload & 8000 bits \\
\hline Data rate & $2.0 \mathrm{Mbps}$ \\
\hline Transport Layer & User Datagram Protocol (UDP) \\
\hline Routing & Ad-hoc On-Demand Distance Vector (AODV) \\
\hline 802.11 model & DEI multirate \\
\hline Tx freq & $2442.0 \mathrm{MHz}$ \\
\hline Tx power & $10.0 \mathrm{dBm}$ \\
\hline Antenna gain & $0.0 \mathrm{~dB}$ \\
\hline Rx noise power & $-69.5 \mathrm{dBm}$ \\
\hline Propagation model & free-space with correlated shadow fading \\
\hline Fading frequency & $1.0 \mathrm{~Hz}, 5.0 \mathrm{~Hz}$ \\
\hline Fading depth & varied \\
\hline MS separation & $7.7 \mathrm{~m}, 12.2 \mathrm{~m}, 17.3 \mathrm{~m}, 21.7 \mathrm{~m}, 24.4 \mathrm{~m}$ \\
\hline
\end{tabular}

2) The complexity reduction attained by the proposed system is evaluated in terms of the number of decoding operations against those of the above-mentioned benchmarkers.

3) Lastly, we investigate the attainable performance of the SLT coded systems when subjected to multi-user interference.

The outline of this paper is as follows. Section II summarizes the characteristics of SLT codes. In Section III, we describe the transmission model utilised and the implementation of the fading channel used to assess the SLT coded system. The details of our simulation results recorded for the SLT coded system in the $802.11 \mathrm{~b}$ networks considered are presented in Section IV, followed by our conclusions.

\section{Systematic Luby TRANSForm Codes}

The conventional LT code [7] was proposed in 2002, which exhibits a higher source packet recovery probability associated with a lower implementation complexity than the family of Fountain codes [8]. However, since they have been proposed for the conventional Ethernet-based Internet, where infinitesimally low BERs prevail, in the presence of the high BERs of wireless channels they suffer from catastrophic packet error propagation. For transmission over wireless channels they have to be combined with classic physical-layer FEC schemes to enhance their performance [9]-[11]. However, the attainable performance improvement remained limited due to the sub-optitmum hard-decisions employed in the LT decoding process. To circumvent this deficiency of conventional LT codes, SLT codes were proposed by Nguyen, Yang and Hanzo [6]. The structure of an SLT code facilitates soft decoding, which may be implemented using a classic belief-propagation technique applied to the code's Tanner graph [12].

In principle, the family of SLT codes is formed by directly concatenating the information bits, known as systematic bits, with the parity bits of the original LT code [7]. This process is illustrated in Fig. 1, where the information bits are partitioned into source blocks before forming both the systematic packets and non-systematic encoded packets. The arrangement allows the information bits to be recovered despite the packet loss caused by fading. Again, the decoding of SLT codes may be performed based on the classic belief-propagation concepts utilised when decoding Low-Density Parity Check (LDPC) codes [13]. The parity bits of the classic LT codes are constructed by selecting the required number of 'packets' to be combined for generating the next LT-coded packet on the basis of the Robust Soliton Degree (RSD) distribution [7]. However, the RSD distribution consists of several single parity check bits, also known as degree-one nodes. Hence, employing the RSD distribution is not particularly suitable for an SLT code utilising iterative decoding. Moreover, incorporating single parity check bits is not essential for an SLT code, since their role is performed by the provision of the original systematic bits. To improve the performance of both LT and SLT codes, the authors of [11], [14] proposed several distributions, namely the Improved RSD (IRSD) and the Truncated Degree (TD) distribution. Both of these distributions reduce the number of degree-one nodes introduced by the encoding process.

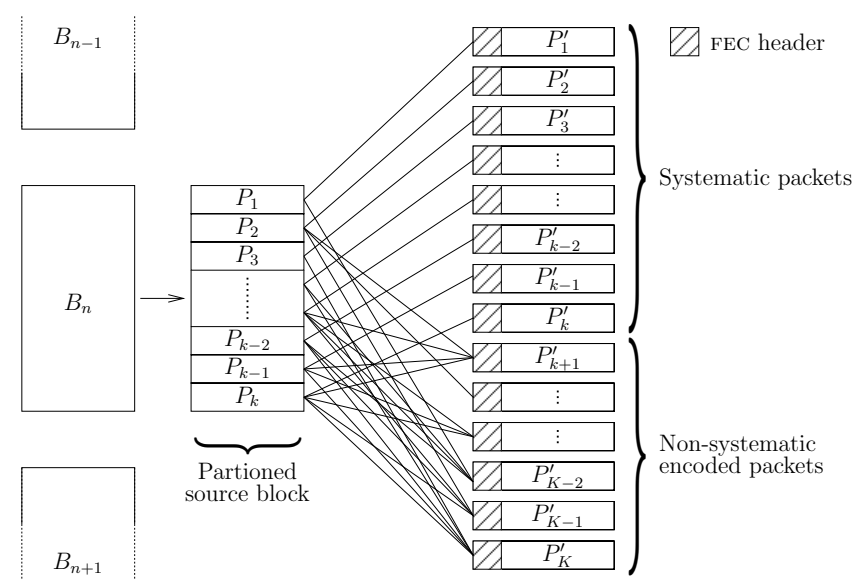

Fig. 1. SLT Encoding Process

The performance of SLT codes using soft decoding was characterised in [6]. In order to investigate the potential of an SLT code further, in this treatise they are applied in the form of an application layer FEC in an 802.11b network model, which is described in detail in the next section.

\section{TRAnSmission Model}

The scenario investigated is comprised of two MSs subject to correlated shadow fading. An ad-hoc network, using the 802.11 model [15], included as part of the NS-2 network simulator [4], was configured as shown in Table I.

Given the low-power indoor scenario considered, we use the typical line-of-sight propagation, which included a realistic shadow fading channel. Simulations were then performed for a number of configurations parameterised by the received signal amplitude, the fading frequency and the standard deviation of the log-normally distributed shadow fading component. Rather than adjusting the transmitter power within the simulation, the received signal amplitude was set by appropriately adjusting the physical distance between the MSs. The fading frequencies where harmonized with the SLT code rate, where the former sets a lower limit on the tolerable shadow fading frequency because when the MS remains semi-permanently shadowed, virtually no amount of application layer FEC coding is capable of recovering the source block. Shadow fading frequencies of $1.0 \mathrm{~Hz}$ and $5.0 \mathrm{~Hz}$ were considered. 
Before the simulations were performed, a reference received signal amplitude, c.f. distance, was selected, which results in a raw Packet Loss Ratio (PLR) of $10^{-6}$ at the receiver, which is the PLR associated with delivering the packets from the transport layer to the application layer, i.e the PLR before the action of the application layer FEC. This reference level was found to occur, when the distance between the MSs was $24.4 \mathrm{~m}$. Additional simulations were performed with the distance between the MSs set to give received signal amplitudes of $1.0 \mathrm{~dB}, 3.0 \mathrm{~dB}, 6.0 \mathrm{~dB}$ and $10.0 \mathrm{~dB}$ above this reference level. The corresponding SINR values were $12.6 \mathrm{~dB}$, $14.6 \mathrm{~dB}, 17.6 \mathrm{~dB}$ and $21.6 \mathrm{~dB}$.

The second part of our study considers the performance of the application layer FEC, when transmissions are subjected to multi-user interference. Again, the primary pair of nodes transfer a real-time stream, optionally involving the application layer FEC, while an additional pair of users is randomly positioned within our ad-hoc network in order to consider the worst-case interference-contaminated scenario. At this time a transaction representing file transfer using FTP is started. The size of the file to be transferred is determined according to the distribution described by Barford [16]. Upon completion of file transfer and after a delay determined by the Poisson distribution [17], the next pair of randomly positioned nodes replace the current pair and transfer their file. This process is replicated for each of the additional pairs of MSs considered in the simulation. As a consequence, the Signal-to-Interferenceand-Noise-power Ratio (SINR) of a specific pair of MSs, in which Node $i$ transmits data to Node $j$, may be expressed as

$$
\begin{aligned}
S I N R^{i j}= & P_{T x}^{i j}-L_{p l}^{i j}-L_{\text {thermal }}-L_{\text {fading }} \\
& -\sum_{l=1, l \neq i, j}^{L} \max \left\{0,\left(P_{T x}^{l j}-L_{p l}^{l j}\right)\right\},
\end{aligned}
$$

where $P_{T x}^{i j}$ and $P_{T x}^{l j}$ represent the transmit powers from Node $i$ and interfering Node $l$ to Node $j$, respectively; $L_{p l}^{i j}$ and $L_{p l}^{l j}$ are the path loss from Node $i$ and interfering Node $l$ to Node $j$, respectively; $L_{\text {thermal }}$ is thermal noise power; and $L_{\text {fading }}$ is fading-induced attenuation. We note that the SINR value is time-variant as a consequence of the fading as well as owing to the effects of Carrier Sense in the 802.11 wireless network [1].

\section{System PERformance}

This section characterises the attainable performance of the system described in Sections II and III. Further benchmark schemes, including plain repeat transmissions, conventional LT codes and RLFCs [8], were provided for comparison. For conveniences, we set a packet size of 8000 bits and vary the code rate between $\mathrm{R}=1 / 2$ and $\mathrm{R}=1$, for all coding schemes.

Fig. 2 shows the PLR of the $802.11 \mathrm{~b}$ system considered, when the MSs are located at various positions and when diverse fading deviations were chosen from a range of values. According to Section III, when the MSs are moved farther apart, the effect of path-loss increases, hence the SINR decreases, while the PLR increases. Observe furthermore from Fig. 2 that for each distance between the MSs considered, there is a critical value of the fading Standard Deviation (SD),

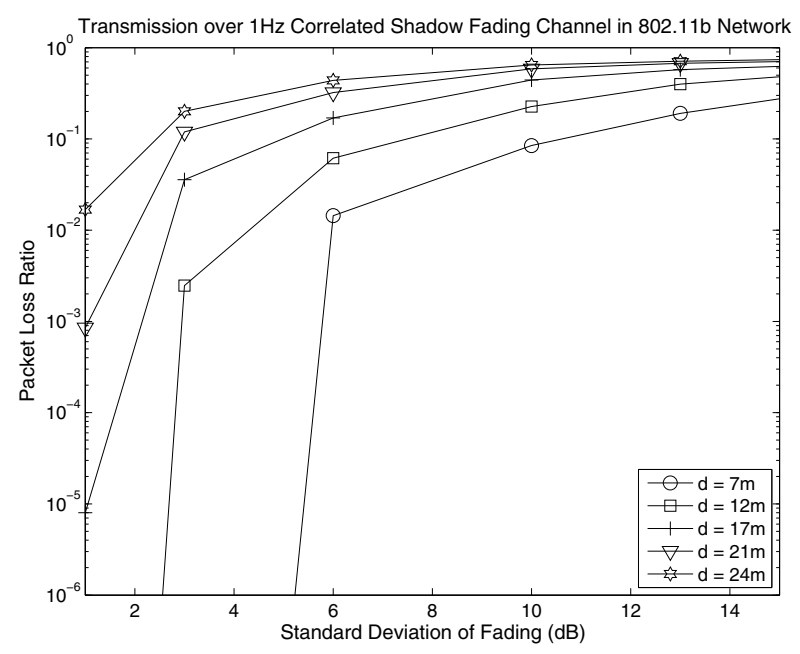

Fig. 2. Packet Loss Ratio of 802.11 b Network when Transmitting over a Correlated Shadow Fading Channel fluctuating at 1Hz: packet_size = 8000 bits, MS distances of $d=\{7,12,17,21,24\} m$.

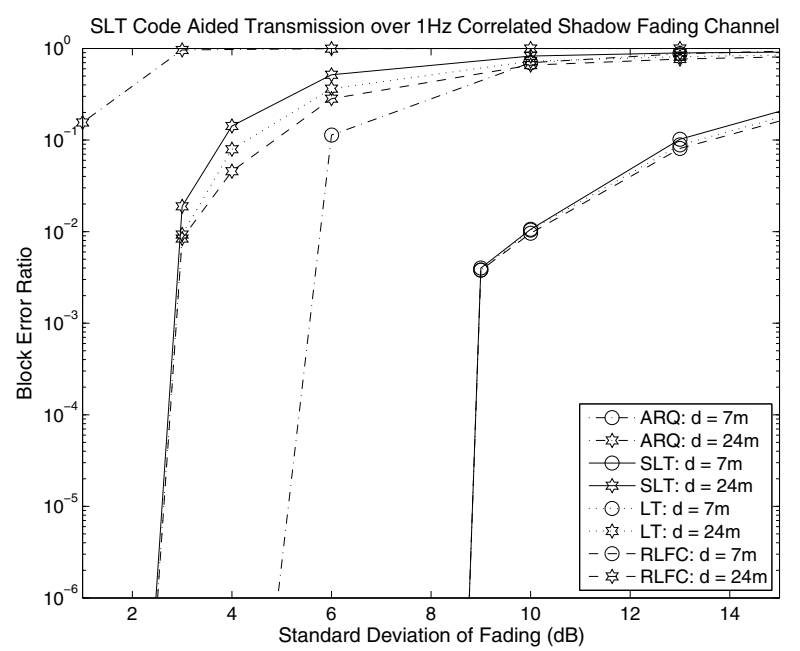

Fig. 3. Block Error Ratio of ARQ Transmission, RLFC Code and SLT Code Aided 802.11b Network when Communicating over a Correlated Shadow Fading Channel fluctuating at 1Hz: packet_size $=8000$ bits, MS distances of $d=\{7,24\} m$.

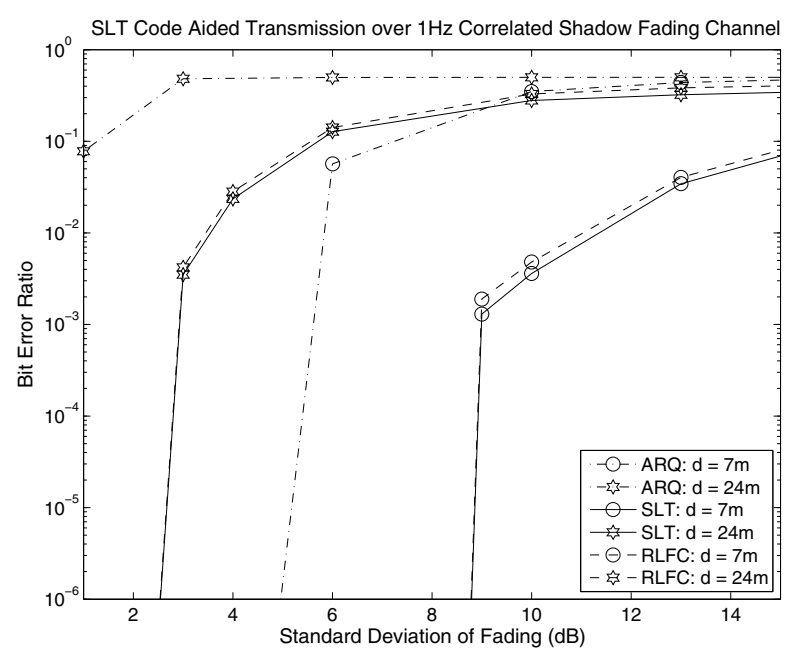

Fig. 4. Bit Error Ratio of ARQ Transmission, RLFC Code and SLT Code Aided 802.11b Network when Communicating over a Correlated Shadow Fading Channel fluctuating at 1Hz: packet_size $=8000$ bits, MS distances of $d=\{7,24\} m$. 
at which the PLR sharply increases. More particularly, when the MSs' distance is $7 \mathrm{~m}$, the critical value of shadow fading SD is about $6 \mathrm{~dB}$, while that value is $3 \mathrm{~dB}$ for the MSs at a distance of $12 \mathrm{~m}$.

The performance of Automatic Repeat Request (ARQ) Type I based transmission [18], of the SLT-coded and LT-coded as well as RLFC coded $802.11 \mathrm{~b}$ networks is characterized in Figs. 3 and 4. Observe in the figures that as expected, all coding schemes outperform the simple ARQ transmission based scheme in terms of both the achievable BER and BLER, where difference between the PLR and BLER was interpreted in Fig. 1. Compared to the LT and RLFC schemes, the SLT code is slightly inferior in term of its BLER, as seen in Fig. 3. However, as illustrated in Fig. 4, it is capable of achieving a lower BER than the RLFC code.

Fig. 5 shows the achievable BER of the SLT code in terms of the distance between the two MSs. As expected, the BER increases, when the MSs' distance is increased. Due to the effect of the critical SD/distance values which gravely affect the PLRs seen in Fig. 2, for example, the BERs seen in Fig. 5 also change substantially when the fading deviation approaches the critical value. More particularly, observe in Fig. 5 that when the MSs are separated by $7 \mathrm{~m}$, the BER drops below $10^{-6}$, when the fading deviation is about $10 \mathrm{~dB}$. When the MS distance is increased to $12 \mathrm{~m}$, the critical value of shadow fading SD becomes $6 \mathrm{~dB}$.

The effect of the shadow fading correlation on the SLT code is characterised in Fig. 6. In our simulations, the shadow fading frequencies of $1 \mathrm{~Hz}$ and $5 \mathrm{~Hz}$ were employed. According to Fig. 6, the SLT code performs better at the higher shadowing frequencies. At the same distance, the SLT code operating in the correlated channel fluctuating at $5 \mathrm{~Hz}$ can tolerate a $3 \mathrm{~dB}$ higher shadow fading SD, compared to the correlated shadowfading channel fluctuating at $1 \mathrm{~Hz}$.

Fig. 7 further characterises the advantage of SLT codes over the benchmark codes. In case of LT and RLFC codes, the rateless decoding operations must be carried out, even when there are no packet loss events. By contrast, a benefit of the systematic bits included in the SLT code is that it only activates the decoding process, when there is a packet loss event in the systematic bits. Thus, it is capable of reducing the decoding complexity imposed when the channel conditions are favourable. In our investigations the SLT coded system did not require any decoding operations, when the shadow fading standard deviation (SD) was less than $3 \mathrm{~dB}$ and the MS separation was $7 \mathrm{~m}$. When the shadow fading SD increased to $10 \mathrm{~dB}$, the number of decoding operations remained about $20 \%$ lower than that of the LT and RLFC coded systems. Hence, the signal processing-related power consumption of the system can be reduced. These characteristics render the SLT code attractive for applications, which require both a low latency and a low power consumption.

Finally, the SLT coded system was investigated in a multiuser environment. The number of users was varied from 2 to 50 in order to quantify the effect of interference imposed by the ubiquitous hidden node problem. In this scenario, the monitored pair of nodes was separated by $24 \mathrm{~m}$ and the other pairs by upto $24 \mathrm{~m}$ with at least one node being within $24 \mathrm{~m}$ of

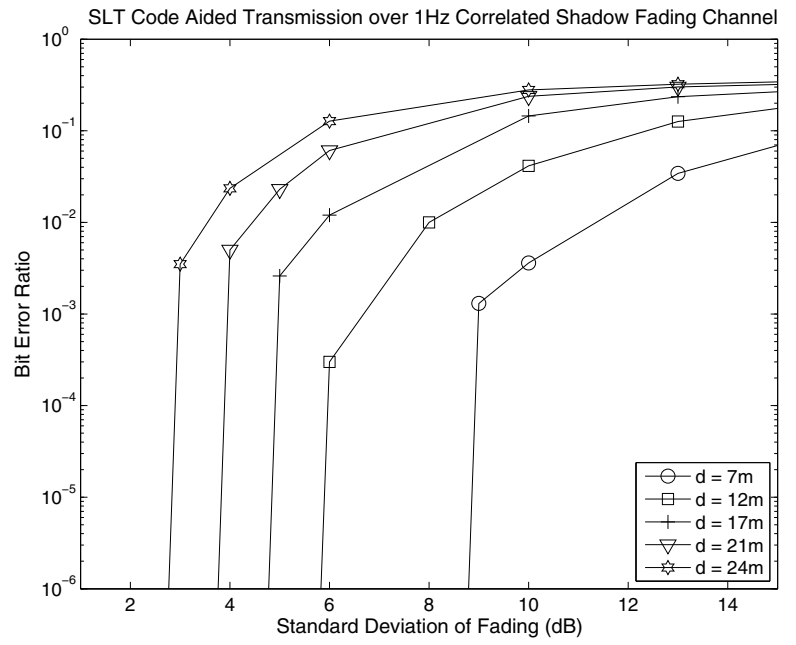

Fig. 5. Bit Error Ratio of the SLT Code Aided 802.11b Network when Communicating over a Correlated Shadow Fading Channel fluctuating at $1 \mathrm{~Hz}$ : packet_size $=8000$ bits, MS distances of $d=\{7,12,17,21,24\} \mathrm{m}$.

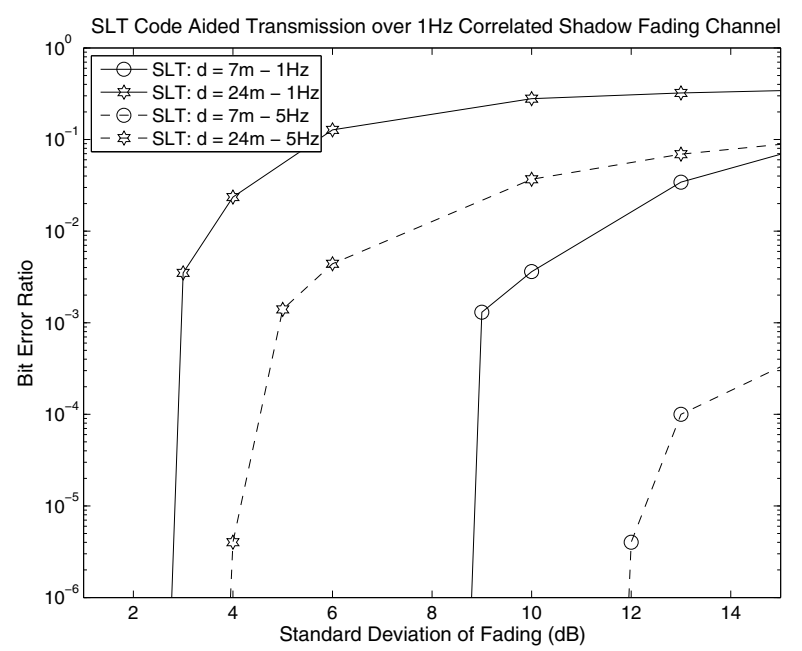

Fig. 6. Bit Error Ratio of the SLT Code Aided 802.11b Network when Communicating over Correlated Shadow Fading Channels fluctuating at $1 \mathrm{~Hz}$ and $5 \mathrm{~Hz}$ : packet_size $=8000$ bits, MS distances of $d=\{7,24\} \mathrm{m}$.

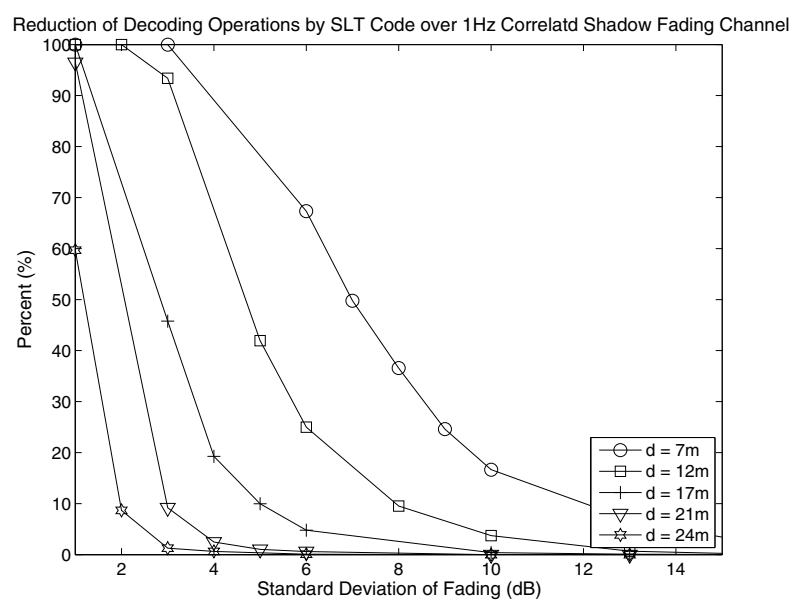

Fig. 7. The Average Percentage of Application-Layer Decoding Operations Reduced by the SLT Code, compared to LT and RLFC codes, in $802.11 \mathrm{~b}$ Network when Communicating over a Correlated Shadow Fading Channel fluctuating at $1 \mathrm{~Hz}$ : packet_size $=8000$ bits, MS distances of $d=$ $\{7,12,17,21,24\} m$. 
one of the monitored pair. According to queuing theory [17], the packet arrival or generation instant of a specific link's transaction is typically modelled by the Poisson exponential distribution. Therefore, the packet arrival is assumed to be Poisson distributed with a mean of 1.0 second. Based on the assumptions stipulated, the average SINR value of the pair of communicating nodes considered is shown in Fig. 8. Clearly, the SINR reduces due to the increased interference by the number of users in the network.

As a consequence of Fig. 8, Fig. 9 characterized the BER performance of the SLT coded system operating in the $802.11 \mathrm{~b}$ multiuser environment. According to Fig. 9, the SLT coded system exhibited a BER below $10^{-5}$, compared to about $1 \%$ for the uncoded scheme when 10 users communicated simultaneously. In case of a large number of communicating users, say 50 users, the BER of the SLT coded system is approximately three times lower than that in the uncoded scenario.

\section{CONCLUSION}

In this paper, we have invoked an SLT code for transmission over the correlated fading channel in order to investigate its performance benefits in the application layer of an $802.11 \mathrm{~b}$ ad-hoc network. The simulation results show that the SLT code provides a comparable performance to the LT and RLFC coded system in terms of its BER and BLER. However, as seen in Fig. 7 the SLT requires a reduced number of decoding operations, compared to the others. More particularly, observe in Fig. 7 when fading deviations are $3 \mathrm{~dB}$ and $10 \mathrm{~dB}$, and the MSs' distance is $7 \mathrm{~m}$, the SLT coded system is capable of reducing the number of decoding operations by $100 \%$ and $20 \%$, respectively. Additionally, as seen in Fig. 6 that the SLT coded system performs better when the shadow fading correlation increases. Finally, as observed in Fig. 9 SLT coded systems may reduce the BER from $10^{-2}$ to below $10^{-5}$, compared to the uncoded scheme when supporting 10 users.

\section{REFERENCES}

[1] "IEEE standard for information technology - telecommunications and information exchange between systems - local and metropolitan area networks - specific requirements. Part 11: Wireless LAN medium access control (MAC) and physical layer (PHY) specifications," 2007.

[2] L. Hanzo, M. Mnster, B. J. Choi, and T. Keller, OFDM and MC-CDMA for Broadcasting Multi-User Communications, WLANs and Broadcasting. Wiley-IEEE Press, 2003.

[3] L. Hanzo, J. S. Blogh, and S. Ni, 3G, HSPA and FDD versus TDD Networking: Smart Antennas and Adaptive Modulation. Wiley, 2nd ed., Feb. 2008

[4] http://www.isi.edu/nsnam/ns, "The ns-2 network simulator."

[5] R. Steele and L. Hanzo, Mobile Radio Communications: Second and Third-generation Cellular and WATM Systems. John Wiley - IEEE Press, 2nd ed., May 1999.

[6] T. D. Nguyen, L. L. Yang, and L. Hanzo, "Systematic Luby transform codes and their soft decoding," in IEEE Workshop on Signal Processing Systems, pp. 67-72, 17-19 Oct. 2007.

[7] M. Luby, "LT codes," in Proceedings of the 43rd Annual IEEE Symposium on Foundations of Computer Science, 2002, pp. 271-280, 16-19 Nov. 2002.

[8] D. J. C. MacKay, "Fountain codes," IEE Proceedings-Communications, vol. 152, pp. 1062-1068, 9 Dec. 2005.

[9] R. Y. S. Tee, T. D. Nguyen, L. L. Yang, and L. Hanzo, "Serially concatenated Luby transform coding and bit-interleaved coded modulation using iteratlive decoding for the wireless Internet," in Proceedings of IEEE 63rd Vehicular Technology Conference, VTC 2006-Spring, vol. 1, pp. 22-26, 7-10 May 2006.

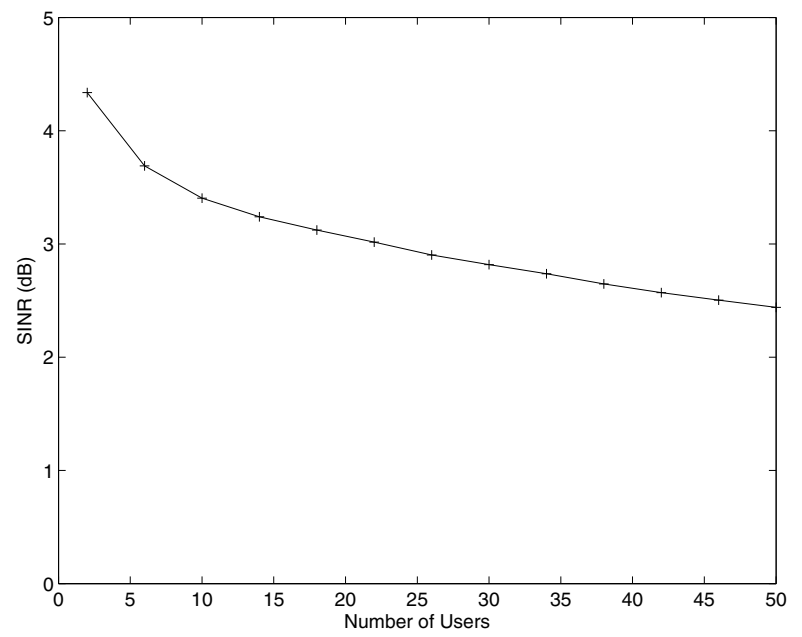

Fig. 8. SINR of the monitored pair of MSs

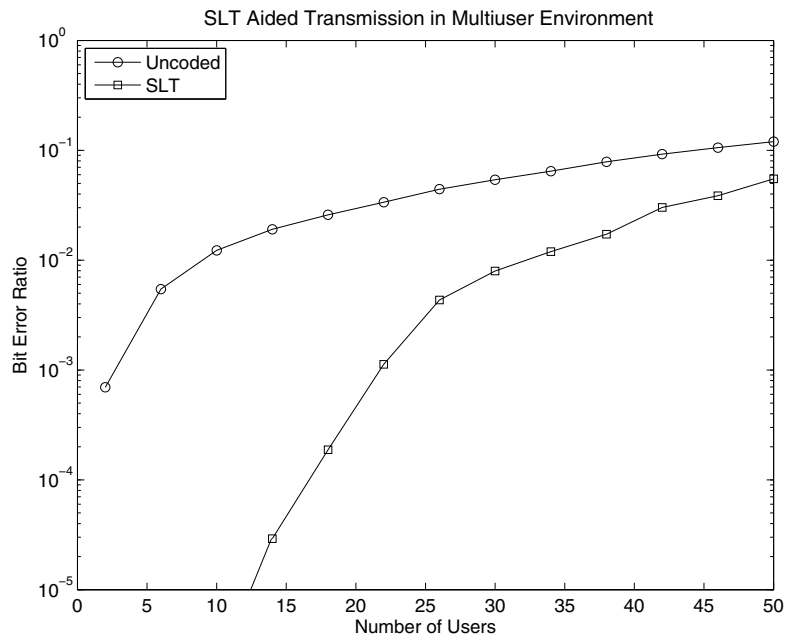

Fig. 9. Bit Error Ratio of SLT Code Aided 802.11b Network when Operating in the Multiuser Environment: packet_size $=8000$ bits, MS distances of $d=24 m$.

[10] R. Y. S. Tee, T. D. Nguyen, S. X. Ng, L. L. Yang, and L. Hanzo, "Luby transform coding aided bit-interleaved coded modulation for the wireless Internet," in Vehicular Technology Conference, VTC-2007 Fall. 2007 IEEE 66th, pp. 2025-2029, Sept. 30 2007-Oct. 32007.

[11] T. D. Nguyen, F. C. Kuo, L. L. Yang, and L. Hanzo, "Amalgamated generalized low density parity check and Luby transform codes for the wireless Internet," in IEEE 65th Vehicular Technology Conference (VTC'07-Spring), pp. 2440-2444, 22-25 April 2007.

[12] R. Tanner, "A recursive approach to low complexity codes," IEEE Transactions on Information Theory, vol. 27, pp. 533-547, Sep 1981.

[13] R. G. Gallager, "Low-density parity-check codes," MA: MIT Press, 1963.

[14] T. Nguyen, L. L. Yang, S. X. Ng, and L. Hanzo, "An optimal degree distribution design and a conditional random integer generator for the systematic Luby transform coded wireless Internet," in IEEE Wireless Communications and Networking Conference (WCNC'08), pp. 243-248, March 31- April 32008.

[15] Department of Engineering and Information, University of Padova, "dei80211mr: a new 802.11 implementation for ns-2." www.dei.unipd.it/wdyn/?IDsezione $=5090$.

[16] P. Barford, A. Bestavros, A. Bradley, and M. Crovella, "Changes in web client access patterns - characteristics and caching implications," World Wide Web, vol. 2, pp. 15-28, 1999.

[17] D. Gross and C. M. Harris, Fundamentals of Queueing Theory. WileyBlackwell, 3rd ed., 1998.

[18] S. Lin and D. J. Costello, Error Control Coding. Prentice Hall, 2nd ed., 2004. 